\title{
Effort thrombosis in an American football player
}

\author{
D.A. Gorard, MRCP \\ Department of Medicine, Queen Mary's University Hospital, London, UK
}

\begin{abstract}
A case of lower limb venous thrombosis occurring in a young male after playing American football is described. The features are compared to the more widely recognized effort thrombosis of the upper limb veins. The possibility of effort thrombosis needs to be considered in athletes presenting with leg injuries.
\end{abstract}

Keywords: Effort thrombosis, American football

\section{Introduction}

Thrombosis of the deep veins of the upper limb after strenuous exercise or trauma is a rare but well recognized condition, often called Paget-Schroetter's syndrome or effort thrombosis ${ }^{1,2}$. A case of thrombosis involving the deep veins of the lower limb in an American football player is described.

\section{Case report}

A previously well 23-year old male was admitted with gradually increasing pain and swelling of the right thigh. He was a non-smoker, on no medication, and in particular there was no history of intravenous drug abuse. He was an enthusiast for American football, and had recently been playing competitively for approximately 10 hours each week. Examination revealed a discoloured and swollen warm right lower limb with dilated superficial veins. There were no other abnormalities, and in particular, examination of the abdomen and rectum was normal.

Lower limb venography confirmed extensive thrombosis occluding the right femoral and iliac veins. Ultrasonography and computerized tomography of the abdomen and pelvis were unremarkable. Haematocrit, platelet count, prothrombin time, activated partial thromboplastin time and antithrombin III activity were all normal. Screening for antinuclear factor and antibodies to cardiolipin was negative. The patient was anticoagulated on admission with intravenous heparin and then warfarin. The swelling of the right lower limb subsided and there were no features to suggest pulmonary embolus.

\section{Discussion}

The term 'effort thrombosis' is usually used in the description of spontaneous primary thrombosis of the axillary and subclavian veins. Such thrombosis is more likely to occur in young adults, and males are affected more frequently than females. In most cases there is a history of recent trauma or unaccustomed strenuous and repetitive exercise involving the affected (usually right) arm. Thus direct or indirect trauma to the vein (possibly causing a tear in the intima), may be an initiating factor in such venous thrombosis as may be dehydration in athletes.

There have now been a few isolated reports of effort thrombosis involving the lower limbs of sportsmen; these relate to the sports of running ${ }^{3}$, skiing ${ }^{4}$ and martial arts training ${ }^{5}$. In the case described, the lower limb veins were involved, but the same aetiological factors found in upper limb effort thrombosis were present, namely strenuous exercise and trauma whilst playing American football.

One other factor may be relevant in this patient's case: participants in American football wear very tight trousers compressing the thighs in order to hold protective thigh pads in position. It is feasible that significant venous stasis might be introduced, particularly during rest periods off the field of play. Such venous stasis would in turn lead to an increased risk of venous thrombosis.

Awareness of the possibility of lower extremity effort thrombosis is needed, so that this differential diagnosis can be considered when patients involved in exercise or sports (both contact and noncontact) present with leg pain or swelling.

\section{Acknowledgement}

I thank Dr C.S. McIntosh for permission to report on this patient.

\section{References}

1 Hughes, E.S.R. Venous obstruction in the upper extremity Br J Surg 1948, 36, 155-163

2 Adams, J.T. and DeWeese, J.A. Effort thrombosis of the axillary and subclavian veins J Trauma 1971, 11, 923-930

3 Harvey, J.S. Effort thrombosis in the lower extremity of a runner Am J Sports Med 1978, 6, 400-402

4 Schobi, R., Kocher, F. and Vorberger, C.H. Tiefe beinvenethrombose beim skisport Schweiz med Wochenschr 1983, 113, 1402-1403

5 Zigun, J.R. and Schneider, S. M. 'Effort Thrombosis' (Paget-Schroetter's syndrome) secondary to martial arts training Am J Sports Med 1988, 16, 189-190 